\title{
OCCURRENCE OF CANDIDA SPECIES COLONIZATION IN A POPULATION OF DENTURE-WEARING IMMIGRANTS
}

\author{
R. CALCATERRA ${ }^{1}$, G. PASQUANTONIO ${ }^{2}$, L.A. VITALI ${ }^{3}$, M. NICOLETTI ${ }^{4}$

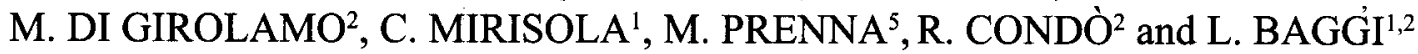 \\ ${ }^{\prime}$ National Institute for Health, Migration and Poverty (NIHMP), Rome, Italy; ${ }^{2}$ Department of \\ Clinical Sciences and Translational Medicine, University of Roma Tor Vergata, Rome, Italy; \\ ${ }^{3}$ School of Pharmacy, Microbiology Unit, University of Camerino, Camerino, Italy; ${ }^{4}$ Department \\ of Biomedical Sciences, University “G. D'Annunzio” Chieti, Italy; ${ }^{5}$ School of Biosciences and \\ Biotechnology University of Camerino, Camerino, Italy
}

Received September 2, 2012 - Accepted November 21, 2012

Infection of the oral cavity and dentures by Candida species are frequent in denture wearers. $C$. albicans is the most common pathogen; however, other emerging Candida species are also responsible for this condition. Few data are available about the occurrence of Candida species in the oral cavities of denture-wearing immigrants to Italy. In this study, we compare the Candida species found in the oral mucosa and on dentures from a population of denture wearing immigrants to Italy to a matched Italian group. Oral swabs were collected from dentures and the underlying mucosa of patients enrolled in the study and were then cultured to test for the presence of Candida species in each sample. Out of 168 patients enrolled (73 Italians and 95 immigrants), 51 Italians $(69.8 \%)$ and 75 immigrants (78.9\%) tested positive for the presence of Candida. Candida albicans was the most frequently observed species overall; however, we found a higher occurrence of $C$. glabrata among immigrants than among Italians. In addition, immigrants displayed a higher incidence of Candida - associated stomatitis and a lower mean age than Candida-positive individuals from the Italian group. Immigrants are more prone to longer colonization of the oral mucosa and dentures by Candida. In these patients, dentures must be checked periodically to prevent the presence of Candida.

The alterations of the oral mucosa by dentures might result from mechanical irritation or inflammatory responses induced by denture materials $(1,2)$. In addition, biofilm formation on the denture surface might contribute to the altered nature of the oral micro-environment among denture wearers (3). Candida species, which comprise $25-50 \%$ of the oral cavity microbiota from healthy individuals and roughly $80 \%$ from denture wearers, are a primary cause of microbial biofilm formation on medical devices (1).

Until recently, Candida albicans was considered the most important opportunistic pathogen in this genus. However, other Candida species, such as Candida glabrata, Candida krusei, Candida parapsilosis and Candida tropicalis, have also emerged as causative agents of infection (1).

Candida glabrata is an emerging fungal pathogen that accounts for $15 \%$ of mucosal and systemic candidoses and is associated with severe

Key words: Candida species, denture-related stomatitis, candidosis

Mailing address: Dr Roberta Calcaterra,

National Institute for Health,

0394-6320 (2013)

Migration and Poverty (INMP)

Via di San Gallicano 25/a

00153 Roma, Italy

Tel.: +3906 58543782 Fax: +390658543686

e-mail: calcaterra@inmp.it reproduced without written permission from the copyright holder. Unauthorized reproduction may result in financial and other penalties DISCLOSURE: ALL AUTHORS REPORT NO CONFLICTS OF INTEREST RELEVANT TO THIS ARTICLE. 
inflammation in denture wearers (1).

Candida dubliniensis has recently been isolated from the oral cavity of human immunodeficiency virus (HIV)-infected patients, leading to its consideration as a novel, emerging, opportunistic pathogen (4). Candida colonization and biofilm formation on dentures may depend on oral hygiene practices, such as overnight denture removal, denture cleanser use, smoking and specific denture characteristics (5).

Denture-related stomatitis (DRS) is an inflammatory process of the mucosa underlying a removable partial or total dental prosthesis or appliance (6). DRS has been reported in more than $60 \%$ of denture wearers, and although it is typically asymptomatic, it occasionally associates with leukoplakia, pseudomembrane formation, erythema and angular cheilitis $(4,6,7)$.

Key factors that can dramatically increase the risk of DRS are loose denture fit, poor denture hygiene and Candida albicans colonization of the denture surface and oral mucosa that contact the denture fitting surfaces (5). Denture materials themselves can contribute to the risk of denture stomatitis, as areas of surface roughness and the hydrophobicity of denture surfaces can promote the attachment of microorganisms and biofilm development $(8,9)$.

The pathogenesis of the Candida-associated denture stomatitis includes local and systemic factors related to the host and to the ability of Candida to adhere and proliferate in the host epithelial tissues (8). Candida-associated denture stomatitis usually occurs when conditions of the oral microenvironment are favorable for the growth and the adhesion of yeast and when systemic factors lead to a systemic immunodepression (10-12).

To date, no data are available about the occurrence of Candida species in the oral cavity of denture-wearing immigrants to Italy. In this study, we compared the colonization of the oral mucosa and dentures from a population of denture-wearing immigrants to Italy with a matched Italian group.

\section{MATERIALS AND METHODS}

\section{Patients}

All denture-wearing patients attending the outpatient department of Social Dentistry Department at National Institutes of Health, Migration and Poverty during the period of June 2011-June 2012 were enrolled. A complete medical and dental history was recorded for each patient, including age, gender, drug use, smoking habits, systemic diseases, DRS symptoms and hygienic habits (modality and frequency) (13).

Patients receiving antifungal or antibacterial treatment within 30 days of enrollment were excluded from the study as well as patients who had undergone immunosuppressive therapies or were affected by an immunosuppressive disease (diabetes, kidney failure, HIV infection). All patients affected with xerostosmy of idiopatic or iatrogenic origin were also excluded.

To identify and characterize the different presentations of DRS, we used the Newton Classification described by Budtz-Jorgensen and Bertram (14):

DRS type I - localised inflammation or hyperaemia points (pin point hyperaemia).

DRS type II - diffuse erythema.

DRS type III - pseudomembrane formation.

\section{Sample collection and isolation}

After examination of the oral cavity, oral swabs were collected from the denture of each patient and from the underlying mucosa according to the procedure described by Marcos-Arias et al. (15). When denture-related stomatitis lesions were evident, a specimen was collected from the lesion. All oral swabs were cultured within $2 \mathrm{~h}$ of collection on CHROMagar Candida medium (Becton Dickinson GmbH, Germany) as well as on Sabouraud dextrose agar plates containing chloramphenicol (Becton Dickinson $\mathrm{GmbH}$, Germany) and were incubated at $37^{\circ}$ $\mathrm{C}$ for $48 \mathrm{~h}$. We considered a Candida-associated denture stomatitis to be an isolation of $>10$ Candida colonies (16).

The plates were scored based on the number of colonies and then subcultured on the same chromogenic medium and Sabouraud dextrose agar to obtain pure cultures.

\section{Characterization of Candida species}

Isolates were identified by conventional mycological methods such as color formation in CHROMagar Candida medium, germ tube tests in calf serum at $37^{\circ} \mathrm{C}$ for 2 days, and microscopic morphology. Additionally, all yeast identified as Candida albicans were screened for their ability to grow at $45^{\circ} \mathrm{C}$ on Sabouraud dextrose agar for 3 days and for chlamydoconidia formation on Casein agar at $30^{\circ} \mathrm{C}$ for 10 days (16).

In these cases, isolates highly suspected to be Candida dublinensis were definitively identified using polymerase chain reaction (PCR). PCR identification of Candida dublinensis with the Candida dublinensis-specific primer

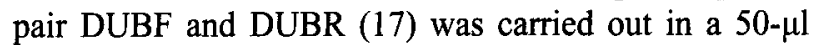
final volume containing $10 \mathrm{pmol}$ each of the forward and reverse primers, $2.5 \mathrm{mM} \mathrm{MgCl} 2,10 \mathrm{mM}$ Tris- $\mathrm{HCl}(\mathrm{pH} 9.0$ at $\left.25^{\circ} \mathrm{C}\right), 10 \mathrm{mM} \mathrm{KCl}, 0.1 \%(\mathrm{vol} / \mathrm{vol})$ Triton X-100, $2.5 \mathrm{U}$ 
of Taq DNA polymerase (Promega), and $25 \mu$ of template DNA-containing cell supernatant. Cycling conditions consisted of $6 \mathrm{~min}$ at $95^{\circ} \mathrm{C}$, followed by 30 cycles of $30 \mathrm{~s}$ at $94^{\circ} \mathrm{C}, 30 \mathrm{~s}$ at $58^{\circ} \mathrm{C}$, and $30 \mathrm{~s}$ at $72^{\circ} \mathrm{C}$, followed by $72^{\circ} \mathrm{C}$ for $10 \mathrm{~min}$. Candida template DNA for use in PCR experiments with the Candida dubliniensis-specific primer pair DUBF (5'GTATTTGTCGTTCCCCTTTC-3') and DUBR (5'-GTGTTGTGTGCACTAACGTC-3') was prepared as described by Donnelly et al. (17).

\section{Statistical analysis}

For the statistical analysis, SPSS software vers. 13 was used (IBM, Armonk, NY, USA). A p $<0.05$ was considered significant.

\section{RESULTS}

We enrolled a total of 190 patients with removable dentures, and 22 were excluded from the study for the following reasons: 5 were affected with diabetes mellitus, 1 was affected with kidney failure, 11 were suffering from xerostomy ( 2 because of Sjogren syndrome and 9 as consequence of anti-hypertensive or anti-depressive therapy) and 5 had received antimycotic or antibiotic therapy within 30 days of enrollment.

Of the remaining 168 patients, 73 were Italians (described as group 1) and 95 were immigrants (described as group 2), who were defined as people born in a country other than Italy that came to Italy within three years of enrollment in the study.

The demographics of the patients in group 1 (Italian patients) and group 2 (immigrant patients) are listed in Table I.

In total, 51 Italians $(69.8 \%)$ and 75 immigrants $(78.9 \%)$ tested positive for contamination by Candida species $(\mathrm{p}=0.12$, chi-squared test $)$. The patients displaying Candida-colonization of the oral mucosa were younger in group 2 than group $1(71.5 \pm 11 \mathrm{vs}$ $49.3 \pm 11.2$ years $\pm \mathrm{ds} \mathrm{p}<0.05$, Student's $t$-test).

Italian patients colonized by Candida species other than albicans had a younger mean age than those colonized by $C$. albicans $(58 \pm 8.1$ vs $75 \pm 9.2$ $\mathrm{p}<0.05$ chi-squared test). Fig. 1 shows the percentage of patients positive for the colonization of dentures and oral mucosa, divided according to Candida species. Table II shows the frequency of Candida species isolated from Italian patients (group 1) and immigrant patients (group 2) based on culture and molecular methods. The most frequently isolated species from oral mucosa and dentures from group 1 were Candida albicans 33 (64.7\%), Candida glabrata 5 (9.8\%), Candida dubliniensis 2 (3.9\%), and Candida tropicalis 2 (3.9\%). From group 2, the most frequently isolated species were Candida albicans 52 (69.3\%), Candida glabrata 10 (13.3\%), Candida dubliniensis 1 (1.3\%), Candida tropicalis 1 $(1.3 \%)$ and Candida krusei 1 (1.3\%).

Simultaneous colonization by two yeast species was found in $6(11.7 \%)$ oral mucosa and denture samples from patients in group 1, particularly Candida albicans and Candida krusei 4 (7.8\%), Candida albicans and Candida parapsilosis 1 $(1.9 \%)$ and Candida albicans and Candida tropicalis $1(1.9 \%)$. In group 2 , we found simultaneous colonization in $13(17.3 \%)$ oral mucosa and denture samples, particularly Candida albicans and Candida glabrata 5 (6.6\%), Candida albicans and Candida krusei 4 (5.3\%) and Candida albicans and Candida tropicalis 4 (5.3\%).

The frequency of Candida albicans colonization

Table I. Demographic characteristic of Italian (group 1) and immigrant (group 2) patients.

\begin{tabular}{|c|c|c|}
\hline & Group 1 & Group 2 \\
\hline Gender (F:M) & $39: 34$ & $40: 55$ \\
\hline Mean age & 69.2 (range 43-86) & 49.8 (range 22-68) \\
\hline
\end{tabular}


Table II. Presence of different species of Candida in positive oral and denture specimens in Italian (group 1) and immigrant ( group 2) patients.

\begin{tabular}{|c|c|c|}
\hline Yeast growth & Group 1 & Group 2 \\
\hline C. albicans & $33(64.7 \%)$ & $52(69.3 \%)$ \\
\hline C. glabrata & $5(9.8 \%)$ & $10(13.3 \%)$ \\
\hline C. dubliniensis & $2(3.9 \%)$ & $1(1.3 \%)$ \\
\hline C. tropicalis & $2(3.9 \%)$ & $1(1.3 \%)$ \\
\hline C. krusei & $0(0 \%)$ & $1(1.3 \%)$ \\
\hline C. albicans + C. krusei & $4(7.8 \%)$ & $4(5.3 \%)$ \\
\hline C. albicans + C. glabrata & $0(0 \%)$ & $5(6.6 \%)$ \\
\hline C. albicans + C. parapsilosis & $1(1.9 \%)$ & $0(0 \%)$ \\
\hline C. albicans + C. tropicalis & $1(1.9 \%)$ & $4(5.3 \%)$ \\
\hline Total & 51 & 75 \\
\hline
\end{tabular}

Table III. Gravity of lesion of oral mucosa in patients of our series resulted positive to colonization by other species than Candida albicans alone and in association.

\begin{tabular}{|c|c|c|}
\hline \multicolumn{3}{|c|}{ Other than Candida Albicans } \\
\hline lesion gravity & Italians & Immigrants \\
\hline I type & 4 & 2 \\
\hline II + III type & $4+1$ & $5+6^{*}$ \\
\hline \multicolumn{3}{|l|}{ Association } \\
\hline lesion gravity & Italians & Immigrants \\
\hline I type & 5 & $3 *$ \\
\hline II + III type & $2+2$ & $3+4 *$ \\
\hline
\end{tabular}

${ }^{*} p<0.05$ calculated by chi square.

of the oral mucosa and dentures was significantly $(\mathrm{p}<0.05)$ higher than that of other Candida species in both groups 1 and 2 . The colonization by Candida glabrata was more frequent among patients from group 2 than those from group $1(\mathrm{p}<0.05)$.

Table III shows the severity of oral lesions (according to the classification in degree 1-3 $\mathrm{p}<$ 0.05 ) observed from the two groups of patients. Immigrants whose dentures and oral mucosa tested positive for Candida species other than Candida, both alone and in association, displayed oral lesions that were clinically more severe (type II and III) 
Table IV. Denture soaking and brushing habits (Panel A) and frequency of denture cleaning (Panel B) in Italians (group 1) and immigrants (group 2).

$\mathbf{A}$

\begin{tabular}{|l|c|c|c|c|}
\hline & \multicolumn{2}{|c|}{ Group 1 } & \multicolumn{2}{c|}{ Group 2 } \\
\hline & $\begin{array}{l}\text { POS } \\
\mathrm{n}=51\end{array}$ & $\begin{array}{c}\text { NEG } \\
\mathrm{n}=22\end{array}$ & $\begin{array}{c}\text { POS } \\
\mathrm{n}=75\end{array}$ & $\begin{array}{c}\text { NEG } \\
\mathrm{n}=20\end{array}$ \\
\hline Brushing only & 10 & 5 & 20 & 5 \\
\hline $\begin{array}{l}\text { Soaking in solution } \\
\text { only }\end{array}$ & 14 & 5 & 24 & 7 \\
\hline Soaking in water only & 13 & 7 & 13 & 5 \\
\hline Brushing and soaking & 13 & 4 & 16 & 2 \\
\hline Nothing & 2 & 1 & 2 & 1 \\
\hline
\end{tabular}

\section{B}

\begin{tabular}{|l|c|c|c|c|}
\hline & \multicolumn{2}{|c|}{ Group 1 } & \multicolumn{2}{c|}{ Group 2 } \\
\hline & POS & NEG & POS & NEG \\
& $\mathrm{n}=51$ & $\mathrm{n}=22$ & $\mathrm{n}=75$ & $\mathrm{n}=20$ \\
\hline$<1$ time / day & 4 & 4 & 49 & 10 \\
\hline 1 time / day & 13 & 7 & 14 & 5 \\
\hline 2 or $>$ times / day & 34 & 11 & 12 & 5 \\
\hline
\end{tabular}

than those of Italian denture wearers with the same colonization.

In Table IV ( $A$ and $B$ ), we describe the oral and denture hygienic habits of the patients included in the study. No difference existed between the groups of patients divided according to the type and frequency of oral and denture cleaning (test $U, p=0.16$ ) and the colonization by Candida species (Mann Whitney, $\mathrm{p}=$ $0.25)$.

\section{DISCUSSION}

Our results show that the mean age of enrolled patients in group 2 was significantly lower than that of patients in group 1. One explanation for this finding is that, generally, immigrant populations primarily consist of young individuals. Despite their younger age, the immigrant group had severe dental problems that could lead to tooth loss and denture placement. Early denture placement in immigrant patients may cause premature, chronic colonization of the denture by Candida species and an early onset of denture stomatitis.

Our data corroborated that Candida albicans is the most frequently isolated species, followed by Candida glabrata, Candida dubliniensis and Candida tropicalis in the oral mucosa as well as on dentures in both group 1 and group 2. We also reported, in accordance with previous studies, that among non-albicans Candida species, the most frequent yeast isolated from the oral mucosa and dentures is Candida glabrata; however, we newly 


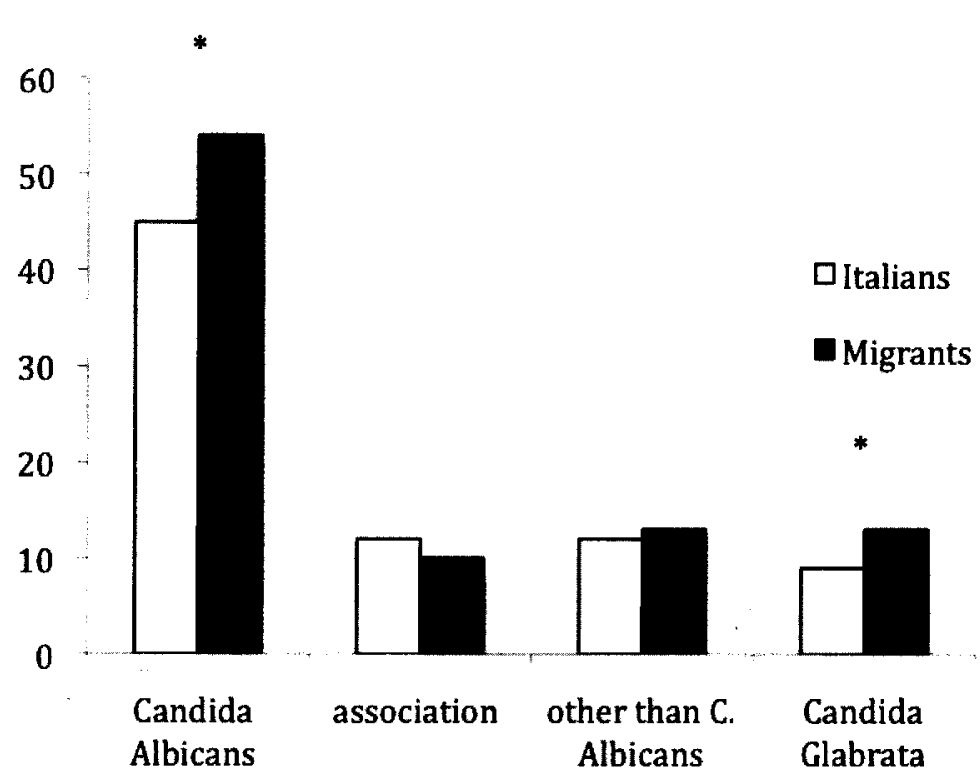

Fig. 1. Percentage of patients positive to the different species of yeast in our series, out of 51 Italians and 75 migrants with a contamination of oral mucosa and dentures. ${ }^{*} p<0.05$ Italians versus immigrants calculated by chi-square test).

report that Candida glabrata is more frequently found in immigrant patients than in native Italians.

Denture use has been demonstrated as a predisposing condition for oral candidiasis $(18,19)$. Compared with Candida albicans, Candida glabrata demonstrated a two-fold greater tendency to adhere to denture acrylic surfaces in vitro (20). With this high propensity to adhere to denture surfaces, it is not surprising that Candida glabrata has been identified as the predominant yeast isolated from dentures of elderly persons with chronic atrophic candidiasis (21). Due to the fact that Candida glabrata is frequently coisolated with other Candida species from oral lesions (22), the role of this organism in pathogenesis is still obscure. Candida glabrata is most frequently coisolated from mucosal lesions with Candida albicans (23). It has been reported that mixed infections with Candida glabrata and Candida albicans can cause more severe symptoms and are more difficult to treat $(23,24)$. Among the clinical manifestations seen in our patients, we found a correlation of Candida species with the type of oral lesion; Candida albicans correlated with the absence of lesions or pin point heperemia/erythema, whereas Candida glabata, Candida dublinesins and Candida tropicalis correlated with pseudomembrane formation. Pseudomembrane formation is likely to be linked to the dramatic histopatological tissue alteration induced by Candida tropicalis, Candida dublinensis and Candida glabrata, as reported previously (26).

Notably, the oral lesions of denture-stomatitis related to non-albicans Candida are clinically more severe in immigrants than in Italians. In our opinion, this finding is due to the fact that immigrants become denture wearers at a younger age, and consequently, the denture is exposed to potential pathogens for a longer period of time. This leads immigrant patients to have more severe denture stomatitis. Additionally, denture materials are often not as reliable as those available in Italy and thus are more prone to colonization.

Moreover, immigrants have limited access to the health care system, and thus, they rarely undergo periodic clinical check-ups of the dentures and the oral mucosa.

We also found that hygienic habits did not impact Candida colonization. This finding differs from previous reports in the literature that describe a 
correlation between hygienic habit and the frequency of oral and denture colonization.

In our opinion, colonization mainly relies upon the denture materials, particularly the micro-porosity of the denture base and the micro-irregularity of the denture surface. These features have a pivotal role in the development of biofilm (26), although hygienic habits might contribute to maintaining the biofilm once established. Further studies evaluating the resistance of different types of resins to different Candida species colonization are required to confirm this assumption.

Interestingly, we found simultaneous colonization by two Candida species in the oldest Italian patients. Therefore, we assumed that age-related immunosuppression may play a major role in the colonization and maintenance of Candida species in the oral cavity as well as in dentures. Several factors, in our opinion, can account for this, such as the chronic use of dentures and the varied composition of oral microbiota and diets.

Because biofilm formation is a risk factor for Candida infection in denture wearers, it is advisable to periodically screen denture wearers for Candida presence. Nevertheless, a prompt treatment is strictly required in patients with non-albicans Candida, as these are the yeast with the highest tendency to invade and destroy the underlying oral mucosa.

\section{REFERENCES}

1. Budtz-Jørgensen E. Oral mucosal lesions associated with the wearing of removable dentures. J Oral Pathol $1981 ; 10: 65-80$.

2. Zomorodian K, Rahimi MJ, Pakshir K, Motamedi M, Ghiasi MR, Rezashah H. Determination of antifungal susceptibility patterns among the clinical isolates of Candida species. J Glob Infect Dis 2011; 3:357-60.

3. Dar-Odeh NS, Shehabi AA. Oral candidosis in patients with removable dentures. Mycoses 2003; 46:187-91.

4. Mosca CO, Moragues MD, Llovo J, Al Mosaid A, Coleman DC, Pontón J. Casein agar: a useful medium for differentiating Candida dubliniensis from Candida albicans. J Clin Microbiol 2003; 41:1259-62.

5. Gendreau L, Loewy ZG. Epidemiology and etiology of denture stomatitis. J Prosthodont 2011; 20:251-60.

6. Dağistan S, Aktas AE, Caglayan F, Ayyildiz A, Bilge M. Differential diagnosis of denture-induced stomatitis, Candida, and their variations in patients using complete denture: a clinical and mycological study. Mycoses 2009; 52:266-71.

7. Fidel PL Jr, Vazquez JA, Sobel JD. Candida glabrata: review of epidemiology, pathogenesis, and clinical disease with comparison to C. albicans. Clin Microbiol Rev 1999; 12(1):80-96.

8. Ramage G, Tomsett K, Wickers BL, et al. Denture stomatitis: a role for Candida biofilms. Oral Surg Oral Med Oral Pathol Oral Radiol Endod 2004; 98:53-59.

9. Tari BF, Nalbant D, Dogruman Al F, Kustimur S. Surface roughness and adherence of Candida albicans on soft lining materials as influenced by accelerated aging. J Contemp Dent Pract 2007; 8:18-25.

10. Yuen HK, Wolf BJ, Bandyopadhyay D, Magruder $\mathrm{KM}$, Salinas CF, London SD. Oral health knowledge and behavior among adults with diabetes. Diabetes Res Clin Pract 2009; 86:239-46.

11. Paillaud E, Merlier I, Dupeyron C, Scherman E, Poupon J, Bories PN. Oral candidiasis and nutritional deficiencies in elderly hospitalised patients. Br J Nutr 2004; 92:861-67.

12. Golecka M, Ołdakowska-Jedynak U, MierzwińskaNastalska E, Adamczyk-Sosińska E. Candidaassociated denture stomatitis in patients after immunosuppression therapy. Transplant Proc 2006; 38:155-56.

13. Kulak-Ozkan Y, Kazazoglu E, Arikan A. Oral hygiene habits, denture cleanliness, presence of yeasts and stomatitis in elderly people. J Oral Rehabil 2002; 29:300-04.

14. Budtz-Jorgensen E, Bertram U. Denture stomatitis. I. The etiology in relation to trauma and infection. Acta Odontol Scand 1970; 28(1):71-92.

15. Marcos-Arias C, Eraso E, Madariaga L, CarrilloMuñozAJ, Quindós G. In vitro activities of new triazole antifungal agents, posaconazole and voriconazole, against oral Candida isolates from patients suffering from denture stomatitis. Mycopathologia 2012; 173:35-46.

16. Marcos-Arias C, Vicente JL, Sahand IH, et al. Isolation of Candida dubliniensis in denture stomatitis. Arch Oral Biol 2009; 54:127-31.

17. Donnelly SM, Sullivan DJ, Shanley DB, Coleman DC. Phylogenetic analysis and rapid identification of Candida dubliniensis based on analysis of ACT1 
intron and exon sequences. Microbiology 1999; 145:1871-82.

18. Ohman SC, Osterberg T, Dahlén G, Landahl S. The prevalence of Staphylococcus aureus, Enterobacteriaceae species, and Candida species and their relation to oral mucosal lesions in a group of 79-year-olds in Göteborg. Acta Odontol Scand 1995; 53:49-54.

19. Lockhart SR, Joly S, Vargas K, Swails-Wenger J, Enger L, Soll DR. Natural defenses against Candida colonization breakdown in the oral cavities of the elderly. J Dent Res 1999; 78:857-68.

20. Luo G, Samaranayake LP, Yau JY. Candida species exhibit differential in vitro hemolytic activities. J Clin Microbiol 2001; 39:2971-74.

21. Wilkieson C, Samaranayake LP, MacFarlane TW, Lamey PJ, MacKenzie D. Oral candidosis in the elderly in long term hospital care. J Oral Pathol Med
1991; 20:13-16.

22. Feng Z, Jiang B, Chandra J, Ghannoum M, Nelson $S$, Weinberg A. Human beta-defensins: differential activity against candidal species and regulation by Candida albicans. J Dent Res 2005; 84:445-50.

23. Redding SW. The role of yeasts other than Candida albicans in oropharyngeal candidiasis. Curr Opin Infect Dis 2001; 14:673-77.

24. Redding SW, Zellars RC, Kirkpatrick WR, et al. Epidemiology of oropharyngeal Candida colonization and infection in patients receiving radiation for head and neck cancer. J Clin Microbiol 1999; 37:3896-900.

25. Sullivan DJ, Moran GP, Coleman DC. Candida dubliniensis: ten years on. FEMS Microbiol Lett 2005; 25:9-17.

26. Ramage G, Martínez JP, López-Ribot JL. Candida biofilms on implanted biomaterials: a clinically significant problem. FEMS Yeast Res 2006; 6:979-86. 